
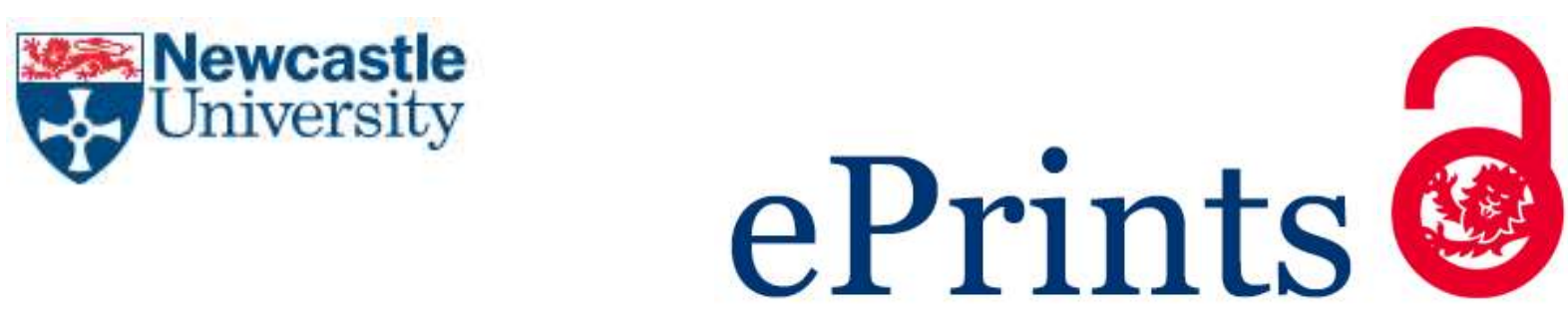

McAllister-Williams RH, Bones K, Goodwin GM, Harrison J, Katona C, Rasmussen J, Strong S, Young AH. Analysing UK clinicians' understanding of cognitive symptoms in major depression: A survey of primary care physicians and psychiatrists. Journal of Affective Disorders 2017, 207, 346-352.

\title{
Copyright:
}

(C) 2017. This manuscript version is made available under the CC-BY-NC-ND 4.0 license

DoI link to article:

http://dx.doi.org/10.1016/j.jad.2016.09.036

Date deposited:

$04 / 07 / 2017$

Embargo release date:

28 September 2017

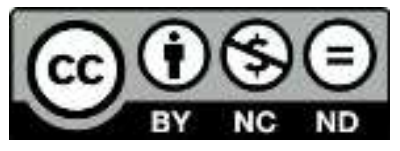

This work is licensed under a

Creative Commons Attribution-NonCommercial-NoDerivatives 4.0 International licence 


\section{Analysing UK clinicians' understanding of cognitive symptoms in major depression: a survey of primary care physicians and psychiatrists}

R Hamish McAllister-Williams ${ }^{\text {a }}$, Kate Bones ${ }^{\text {b }}$, Guy M Goodwin ${ }^{\text {c }}$, John Harrison d, Cornelius Katona ${ }^{\mathrm{e}}$, Jill Rasmussen ${ }^{\mathrm{f}}$, Sarah Strong ${ }^{\mathrm{g}}$, Allan H Young ${ }^{\mathrm{h}, *}$

${ }^{a}$ Newcastle University, Newcastle, UK

${ }^{b}$ Sussex Partnership NHS Foundation Trust, Worthing, UK

${ }^{c}$ Oxford University, Oxford, UK

${ }^{d}$ VU University Medical Center, Amsterdam, the Netherlands

${ }^{e}$ University College London, London, UK

${ }^{f}$ Lingfield, Surrey, UK

${ }^{g}$ Depression Alliance, Croydon, $U K$

${ }^{h}$ King's College London, London, UK

* Correspondence to: Professor Allan H Young, Director, Centre for Affective Disorders, Department of Psychological Medicine, Institute of Psychiatry, Psychology and Neuroscience, King's College London, PO72, De Crespigny Park, Denmark Hill, London, SE5 8AF, UK. Tel.: 0207848 5895; Fax: 02078480298.

E-mail address: allan.young@kcl.ac.uk 


\section{ABSTRACT}

Background: Cognitive dysfunction occurs in depression and can persist into remission. It impacts on patient functioning but remains largely unrecognised, unmonitored and untreated. We explored understanding of cognitive dysfunction in depression among UK clinicians.

Methods: A multi-step consultation process. Step 1: a multi-stakeholder steering committee identified key themes of burden, detection and management of cognitive dysfunction in depression, and developed statements on each to explore understanding and degree of agreement among clinicians. Step 2: 100 general practitioners (GPs) and 100 psychiatrists indicated their level of agreement with these statements. Step 3: the steering committee reviewed responses and highlighted priority areas for future education and research.

Results: There was agreement that clinicians are not fully aware of cognitive dysfunction in depression. Views of the relationship between cognitive dysfunction and other depressive symptom severities was not consistent with the literature. In particular, there was a lack of recognition that some cognitive dysfunction can persist into remission. There was understandable uncertainty around treatment options, given the current limited evidence base. However, it was recognised that cognitive dysfunction is an area of unmet need and that there is a lack of objective tests of cognition appropriate for depressed patients that can be easily implemented in the clinic.

Limitations: Respondents are likely to be 'led' by the direction of the statements they reviewed. The study did not involve patients and carers. 
Conclusions: UK clinicians should undergo training regarding cognitive dysfunction in depression, and further research is needed into its assessment, treatment and monitoring.

Keywords:

Cognitive dysfunction

Depression

Clinician awareness 
Abbreviations: GP, general practitioner; HCP, health-care professional 


\section{Introduction}

Cognitive dysfunction is an important aspect of depression that includes problems with thinking, concentration and memory (American Psychiatric Association, 2013; Hammar and Årdal, 2009). Cognitive dysfunction is highly prevalent in people with depression and has a significant impact on functioning (Jaeger et al., 2006; McIntyre et al., 2013; Shilyansky et al., 2016). In a recent, cross-sectional, observational study in South Korea, greater functional disability and impairment in daily activities, including worse work-related productivity outcomes, were associated with more severe perceived cognitive dysfunction (Kim et al., 2016). The domains of executive function, working memory, episodic memory, attention and psychomotor processing speed are commonly affected in people with depression (McIntyre et al., 2013; Shilyansky et al., 2016), with poorer performance in neuropsychological tests being in the order of small (0.2) to medium (0.5) effect sizes compared to healthy individuals (Lee et al., 2012). Cognitive problems may also remain as residual symptoms during periods of remission from major depressive episodes (Conradi et al., 2011; McClintock et al., 2011), with residual symptoms increasing the risk of relapse (Judd et al., 1998). In a systematic review and meta-analysis of cognitive function, significant moderate deficits in executive function and attention (effect sizes ranging from -0.52 to -0.61 ) were found to persist in patients in whom depressive symptoms had remitted (Rock et al., 2014). This has been confirmed in a recent, large $(n=>700$ completers), randomised trial of three antidepressants (escitalopram, sertraline and venlafaxine), which showed that even in patients whose depression remitted with treatment, there was no significant improvement in cognition, which remained impaired compared to matched healthy control individuals (Shilyansky et al., 2016). 
It has been suggested that cognitive dysfunction in depression is largely unrecognised and unmonitored by clinicians (Greer et al., 2010).

We aimed to explore the understanding of aspects of cognitive dysfunction in clinical depression (major depressive disorder) in the UK, using a multi-step consultation process involving primary and secondary care clinicians with an interest in depression. It was intended that through this process, priority areas to be addressed through education would be highlighted.

\section{Methods}

Using a multi-step process, we obtained and evaluated the views of primary and secondary care experts in depression. In Step 1, a multi-stakeholder steering committee (including eight psychiatrists, psychologists, primary care physicians, and representatives from occupational therapy and a depression charity) provided the three key themes, which were burden, detection and management of cognitive dysfunction in depression. Through round-table discussion, the steering committee developed 10-15 statements for each of these areas to explore the understanding of clinicians and the level of agreement among them.

In Step 2, these statements formed an online survey (hosted by medeConnect, part of Doctors.net.uk) completed by 200 health-care professionals (HCPs) (100 general practitioners [GPs] and 100 psychiatrists) with an involvement in the management of depression in the UK. In order to be included on the HCP panel, participants had to have seen or treated a minimum number of patients with major depressive disorder in the previous 3 months: GPs $\geq 10$ patients; psychiatrists $\geq 20$ patients. Participants were asked to rate their level of agreement with the statements as "strongly disagree", 
“disagree”, “agree”, “strongly agree” or “don’t know/uncertain”. Participants also had the ability to add free-text comments to expand on their responses. The level of agreement among respondents was defined by the steering committee as follows: High agreement, $>66 \%$ of respondents; Very high agreement, $>90 \%$ of respondents.

In Step 3, the steering committee reviewed feedback from Step 2 and highlighted priority areas for future education and research.

\section{Results}

A total of 200 respondents from across the UK completed the questionnaire (Table 1). In addition to rating the proposed statements, some respondents provided specific comments (Supplementary Table S1). Overall, there was High/Very high agreement among GPs and psychiatrists for 10 of 13 statements related to burden of cognitive dysfunction in depression, seven of nine statements on detection and seven of 12 statements on management.

\subsection{Burden}

There was High/Very high agreement from both GPs and psychiatrists for all except three of the statements related to burden of cognitive dysfunction in depression (Fig. 1). This suggested that both GPs and psychiatrists recognise that cognitive dysfunction occurs in depression and can have a significant impact on a patient's life, impairing a person's occupational, social, marital and parental functioning, and reducing their confidence. Additionally, clinicians agreed that cognitive dysfunction contributes to an overall increased economic and social burden of depression on the individual, their family and society as a whole. These views are illustrated by one psychiatrist who commented, "In clinical practice, I have seen the impact of cognitive 
dysfunction on patients' lives and rightly so, it is disabling". There was also agreement that more research into the burden of cognitive dysfunction in depression is required.

However, there was less agreement among clinicians regarding the persistence of cognitive dysfunction and the relationship with depressive symptoms, with views being inconsistent with the evidence that cognitive dysfunction persists into remission, at least in some patients (Rock et al., 2014; Shilyansky et al., 2016). Low agreement ( $\leq 50 \%$ agreement) was reached on Statement 2 (Cognitive dysfunction in depression is independent of depressive symptoms); 50\% of GPs and 57\% of psychiatrists disagreed with this statement, with GP comments including, "How can you have cognitive dysfunction in depression without depression?" and "I've found some patients with "chronic depression" often have associated impaired or reduced cognitive function. I don't know which causes which". Similarly, only $40 \%$ of GPs and $54 \%$ of psychiatrists agreed with Statement 11 (More than $30 \%$ of patients experience persistent cognitive symptoms despite remission of depressive symptoms). Individual comments included, "I associated cognitive dysfunction with the depressive illness but generally expect this to be restored once the patient has recovered from depression", "Usually cognitive function improves in my patients, though not necessarily right at the outset of the improvement in mood" and "I don't routinely see cognitive dysfunction persisting after symptomatic remission. If I did, I'd be investigating them for an independent cognitive problem".

Despite High agreement from psychiatrists, only 52\% of GPs agreed with Statement 12 (Recovery of cognitive function is less likely following repeated episodes of depression). 


\subsection{Detection}

There was High/Very high agreement from both GPs and psychiatrists for seven of the nine statements (Fig. 2). Despite the responses above, there was also agreement that a lack of awareness of cognitive dysfunction in depression among clinicians is an issue. Additionally, there was high agreement between psychiatrists that cognitive dysfunction is poorly understood (79\%), and almost two-thirds of GPs (62\%) agreed with Statement 15 (Cognitive dysfunction in depression is poorly understood by health-care professionals). Despite Very high agreement from psychiatrists, over half of the GPs (54\%) also disagreed that Cognitive dysfunction in depression is more likely to be detected in secondary care (e.g. by psychiatrists) than in primary care (e.g. by GPs). There was high agreement among all clinicians that cognitive dysfunction in depression remains an unmet need and that there is a lack of available tools to detect it, with it usually being assessed simply subjectively. As a GP commented, "I often diagnose depression in patients with memory impairment or other cognitive difficulties. This is largely based on history rather than a formal objective assessment". Concerns raised by psychiatrists included, "There are no systematic ways of assessing cognitive function other than referral for neuropsychology testing, which is not feasible given current resources". Clinicians felt that routine assessment of cognition should be available and that research is needed to assess the cost-effectiveness of such screening. Finally, there was Very high agreement among both GPs and psychiatrists that depression should be considered as a possible diagnosis in elderly patients presenting with mild cognitive dysfunction. 


\subsection{Management}

Management of cognitive dysfunction in depression was the area of biggest disagreement among clinicians, perhaps not surprisingly. There was High/Very high agreement from GPs and/or psychiatrists for just seven of the 12 statements (Fig. 3). Overall, there was high agreement that there is uncertainty around best practice for the management of cognitive dysfunction. This was consistent with the responses to Statements 24, 25, 26 and 27, which related to the evidence for the effects of cognitive behavioural therapy, cognitive training/behavioural activation, exercise or lifestyle changes on cognitive function in depression, respectively, with $28-51 \%$ of clinicians responding “Don’t know/uncertain”. With regard to Statement 28 (Improvement in cognitive dysfunction does not occur with all antidepressants), $60 \%$ of GPs and $65 \%$ of psychiatrists agreed, and there was High agreement among psychiatrists, though not quite with GPs, that recovery of cognitive function does not always occur despite clinical recovery from depression (Statement 31), in line with the evidence (Shilyansky et al., 2016) but not entirely consistent with clinicians' earlier responses to the statements on burden of cognitive dysfunction. Nevertheless, there was High agreement among all clinicians that persistent cognitive dysfunction may be a risk factor for relapse. There was High agreement that research into the effects of antidepressants on cognitive dysfunction should be prioritised, and that the degree of dysfunction should be monitored during long-term follow-up and after antidepressants are discontinued.

\section{Discussion}

This report provides insight into the level of understanding of cognitive dysfunction in depression by GPs and psychiatrists in the UK. Responses of clinicians to the 
statements they were presented with identify a number of areas that need to be addressed in the education of clinicians and/or in research.

While there was High agreement among both GPs and psychiatrists that they recognised cognitive dysfunction in depression and its implications for individuals and society, a concern is that this was simply due to them, in effect, answering leading questions, since they also appear to hold the view that clinicians in general are unaware of this issue. If this latter perspective is correct, then there is a clearly identified educational need for clinicians - both GPs and psychiatrists - regarding the presence and nature of cognitive dysfunction in depression. Appropriate continuing professional development materials should be made available to address the limitations in clinician knowledge and it would be advantageous for GPs and psychiatrists to learn together, allowing both groups to benefit from each other's perspective and different clinical insights and experiences.

Perhaps one of the clearest findings, reflected in the response to a number of statements, is that there is either uncertainty regarding the relationship between depressive symptom severity and cognitive dysfunction, or a belief that when symptoms such as low mood resolve, so does cognitive dysfunction. While the wording of Statement 2 (Cognitive dysfunction in depression is independent of depressive symptoms) was potentially ambiguous in the way it could have been interpreted by the clinician panel, evidence from the largest single study in the literature suggests that the severity of the depression and of the cognitive dysfunction are not correlated (Shilyansky et al., 2016). Cognitive dysfunction may be long lasting after symptom recovery, at least in some patients (Hammar and Årdal, 2009; Rock et al., 2014). Additionally, cognitive dysfunction may pre-date the appearance 
of mood symptoms (Foland-Ross and Gotlib, 2012), with a study of 708 nondepressed individuals highlighting that low episodic memory performance constituted a risk of depression diagnosis 3 years later (Airaksinen et al., 2007). Similarly, despite the uncertain responses to Statement 11 by both GPs and psychiatrists (More than $30 \%$ of patients experience persistent cognitive symptoms despite remission of depressive symptoms), there is clear evidence from a number of studies that cognitive dysfunction persists into remission for significant numbers of patients (Conradi et al., 2011; Jaeger et al., 2006; McIntyre et al., 2013). For example, in a 3-year prospective study of 267 primary care patients, cognitive impairment was present $\sim 94 \%$ of the time during depressive episodes and $44 \%$ of the time during periods of remission (Conradi et al., 2011). Furthermore, recurrent depression has increased detrimental effects on cognitive ability (Baune et al., 2010). Indeed, psychiatrists were more inclined to recognise that recovery of cognitive function is less likely following repeated episodes of depression than GPs, perhaps as a result of differences in how GPs and psychiatrists follow up patients.

There was agreement among both GPs and psychiatrists that cognitive dysfunction in depression is an unmet need and an acknowledgement that more research is required, in relation to both the nature and treatment of the dysfunction. How much this resulted from clinicians being prompted in this direction by the statements is uncertain.

GPs and psychiatrists appeared to have differing views on whether cognitive dysfunction is more likely to be detected in primary or secondary care, which is perhaps not surprising given the potential for response bias inherent in the wording of this particular statement, but, overall, both groups agreed that this remains an area of 
unmet need and there is a lack of readily available tools for assessment and monitoring of cognition. Specific comments from GP and psychiatrist participants included the following: "It's under-recognised and difficult to measure", "Although poor concentration is a core symptom of depression which is often enquired about, other cognitive domains are not commonly tested for/addressed" and "Validated tools for use in primary care would be useful in assessing/detecting and then monitoring cognitive function. The only tools we have are designed primarily for dementiarelated illness".

Instruments exist to assess the level of cognitive impairment in patients with dementia (e.g. Cambridge Cognitive Examination, Mini Mental State Examination); however, these are not fully validated for use in patients with depression. Although subjective assessments are available, such as patient-reported questionnaires, and simple questions on daily function may be asked by the physician, these do not provide as full a picture of cognitive function as more comprehensive objective testing. Furthermore, there is evidence of a lack of correlation between subjective and objective assessment of cognition in patients with depression (Svendsen et al., 2012). Ideally, the cognitive function of a patient with depression would be assessed shortly after diagnosis, both subjectively and objectively, and this would subsequently be monitored during the course of treatment.

At present, there is a clinical tool under development that may address some of these points. The THINC-it tool is quick and easy to use and combines objective and subjective measures. It is capable of identifying cognitive dysfunction in primary and secondary care (McIntyre, 2015). The tool is currently being validated in a large, real-world study. 
A few of the individual comments (Supplementary Table S1) suggested that some doctors were unsure if routine cognitive assessment was appropriate, as tests may be too strenuous and detrimental to a patient's confidence. However, the opposite view may also apply, such that patients are reassured that cognitive impairment identified by the tests is something that is common in depression.

Almost all respondents agreed that depression should be considered as a possible diagnosis in older patients with mild cognitive problems, which may mean that recent educational initiatives on this topic in the UK have been successful. One GP noted, "In early dementia, MDD has high prevalence".

Uncertainty regarding the role of routine assessment of cognitive assessment in depression is further compounded by uncertainty about its best-practice management. Respondents agreed that there is uncertainty about the best-practice management of cognitive dysfunction in patients with depression, which was also apparent in the mixed responses to several of the statements on this topic. The widespread uncertainty among both GPs and psychiatrists about some psychological therapies may be a consequence of a genuine lack of knowledge and/or the low quality of studies in this area, such as the lack of placebo-controlled trials. It was concluded from a meta-analysis of cognitive bias modification interventions in anxiety and depression that effects may be small and not clinically relevant, and that trials are small and of low quality (Cristea et al., 2015). In addition, publication bias was raised as an issue and it was highlighted that many positive outcomes are driven by extreme outliers (Cristea et al., 2015). Stronger evidence is available on the use of cognitive behavioural therapy for the treatment of depression, and a recent meta-analysis considered comparative benefits/harms of cognitive behavioural therapies and second- 
generation antidepressants in initial treatment of major depressive disorder (Amick et al., 2015). Studies on the effects of antidepressants on cognitive dysfunction in depression have also had limitations, with early studies often including only small numbers of patients, not having cognitive function as a primary end point, lacking a placebo control or being restricted to elderly patients. One psychiatrist noted, "In the absence of evidenced treatments, assessments (even if they were available) are difficult to prioritise. Which is a shame".

\section{Limitations}

The statements were reviewed by a relatively small sample size of 200 UK-only respondents and no formal statistical analysis was performed on the results. A full Delphi process for gaining consensus was not employed. By their very nature, statements may lead clinicians, with them perhaps being more likely to agree than disagree. Overall, there were far more "agree" answers than "disagree" or even “don't know" responses. Furthermore, since the survey instrument had not been empirically validated, there may have been significant variability in how individual respondents interpreted the meaning of the statements. Respondents were required to have seen or treated a minimum number of patients with major depressive disorder in the previous 3 months but no information on patient age was collected. The study did not involve patients and carers, who may hold very different perspectives on the topic.

\section{Conclusions}

Overall, these responses from UK GPs and psychiatrists support the notion that UK clinicians should undergo training regarding the presence and nature of cognitive dysfunction in depression, and its relationship to other depressive symptoms. There is 
a need for tools to facilitate the objective assessment of cognition that can be easily used in the clinic, both in primary and secondary care, and education of clinicians regarding the use of such tools. Ongoing research is needed regarding possible treatments for cognitive dysfunction, both physical treatments such as drugs and psychological treatments. The arrival of effective treatments will also lead to the need for research into the cost-effectiveness of screening patients with depression for cognitive dysfunction. 


\section{References}

Airaksinen, E., Wahlin, A., Forsell, Y., Larsson, M., 2007. Low episodic memory performance as a premorbid marker of depression: evidence from a 3-year follow-up. Acta Psychiatr. Scand. 115, 458-465.

American Psychiatric Association, 2013. Diagnostic and Statistical Manual of Mental Disorders. Fifth Edition. DSM-5. American Psychiatric Publishing, Washington DC.

Amick, H.R., Gartlehner, G., Gaynes, B.N., Forneris, C., Asher, G.N., Morgan, L.C., Coker-Schwimmer, E., Boland, E., Lux, L.J., Gaylord, S., Bann, C., Pierl, C.B., Lohr, K.N., 2015. Comparative benefits and harms of second generation antidepressants and cognitive behavioral therapies in initial treatment of major depressive disorder: systematic review and meta-analysis. BMJ 351, h6019.

Baune, B.T., Miller, R., McAfoose, J., Johnson, M., Quirk, F., Mitchell, D., 2010. The role of cognitive impairment in general functioning in major depression. Psychiatry Res. 176, 183-189.

Conradi, H.J., Ormel, J., de Jonge, P., 2011. Presence of individual (residual) symptoms during depressive episodes and periods of remission: a 3-year prospective study. Psychol. Med. 41, 1165-1174.

Cristea, I.A., Kok, R.N., Cuijpers, P., 2015. Efficacy of cognitive bias modification interventions in anxiety and depression: meta-analysis. Br. J. Psychiatry 206, 7-16. 
Foland-Ross, L.C., Gotlib, I.H., 2012. Cognitive and neural aspects of information processing in major depressive disorder: an integrative perspective. Front. Psychol. 3, 489.

Greer, T.L., Kurian, B.T., Trivedi, M.H., 2010. Defining and measuring functional recovery from depression. CNS Drugs 24, 267-284.

Hammar, Å., Årdal, G., 2009. Cognitive functioning in major depression - a summary. Front. Hum. Neurosci. 3, 26.

Jaeger, J., Berns, S., Uzelac, S., Davis-Conway, S., 2006. Neurocognitive deficits and disability in major depressive disorder. Psychiatry Res. 145, 39-48.

Judd, L.L., Akiskal, H.S., Maser, J.D., Zeller, P.J., Endicott, J., Coryell, W., Paulus, M.P., Kunovac, J.L., Leon, A.C., Mueller, T.I., Rice, J.A., Keller, M.B., 1998. Major depressive disorder: a prospective study of residual subthreshold depressive symptoms as predictor of rapid relapse. J. Affect. Disord. 50, 97-108.

Kim, J.M., Chalem, Y., di Nicola, S., Hong, J.P., Won, S.H., Milea, D., 2016. A cross-sectional study of functional disabilities and perceived cognitive dysfunction in patients with major depressive disorder in South Korea: The PERFORM-K study. Psychiatry Res. 239, 353-361.

Lee, R.S.C., Hermens, D.F., Porter, M.A., Redoblado-Hodge, M.A., 2012. A metaanalysis of cognitive deficits in first-episode major depressive disorder. J. Affect. Disord. 140, 113-124.

McClintock, S.M., Husain, M.M., Wisniewski, S.R., Nierenberg, A.A., Stewart, J.W., Trivedi, M.H., Cook, I., Morris, D., Warden, D., Rush, A.J., 2011. Residual 
symptoms in depressed outpatients who respond by $50 \%$ but do not remit to antidepressant medication. J. Clin. Psychopharmacol. 31, 180-186.

McIntyre, R., 2015. The first validated screener for objective and subjective cognitive dysfunction in depression. Eur. Neuropsychopharmacol. 25 (Suppl 2), S669.

McIntyre, R.S., Cha, D.S., Soczynska, J.K., Woldeyohannes, H.O., Gallaugher, L.A., Kudlow, P., Alsuwaidan, M., Baskaran, A., 2013. Cognitive deficits and functional outcomes in major depressive disorder: determinants, substrates, and treatment interventions. Depress. Anxiety 30, 515-527.

Rock, P.L., Roiser, J.P., Riedel, W.J., Blackwell, A.D., 2014. Cognitive impairment in depression: a systematic review and meta-analysis. Psychol. Med. 44, 2029-2040.

Shilyansky, C., Williams, L.M., Gyurak, A., Harris, A., Usherwood, T., Etkin, A., 2016. Effect of antidepressant treatment on cognitive impairments associated with depression: a randomised longitudinal study. Lancet Psychiatry 3, 425-435.

Svendsen, A.M., Kessing, L.V., Munkholm, K., Vinberg, M., Miskowiak, K.W., 2012. Is there an association between subjective and objective measures of cognitive function in patients with affective disorders? Nord. J. Psychiatry 66, 248-253. 


\section{Table 1}

Role and geographical location of HCP panel.

GPs

Psychiatrists

Role

Seniority GP, \%

GP partner/Principal

Salaried GP

GP with special interest in mental health, \%

Seniority psychiatrist, \%

Consultant

Associate specialist

Staff grade

Specialist registrar 4 
Psychiatry sub-speciality, \%

General adult psychiatrist

Old age psychiatrist

Mean major depressive disorder caseload in previous 3 months, $n$

Region, $\mathbf{n}$

North West

North East

Yorks and Humber

East Midlands

West Midlands

Eastern England

London 
South Central

South West

Scotland

Wales

Northern Ireland

Abbreviations: HCP, health-care practitioner; GP, general practitioner. 


\section{Supplementary Table S1}

Individual comments received from GPs and psychiatrists.

GP comments

Psychiatrist comments

\section{Burden}

Further research in every aspect of clinical

In older adults, it can be difficult to

medicine is always a welcome development

distinguish between cognitive dysfunction in

depression and cognitive impairment

How can you have cognitive dysfunction in

In clinical practice, I have seen the impact of depression without depression?

cognitive dysfunction on patients' lives and

rightly so, it is disabling

I think this is poorly recognised especially if I don't routinely see cognitive dysfunction

we just use the PHQ9 as a scoring tool

persisting after symptomatic remission. If I

without looking at the individual responses

did, I'd be investigating them for an

independent cognitive problem

Usually cognitive function improves in my

Sometimes the cognitive dysfunction seen in

patients, though not necessarily right at the

depression may be a precursor symptom of

outset of the improvement in mood

underlying dementia

I associated cognitive dysfunction with the

Frequently, elderly individuals are referred

depressive illness but generally expect this to

by their GP for assessment of poor memory,

be restored once the patient has recovered

which, after full assessment, is found to be

from depression

secondary to depression. Following adequate

treatment, there usually appears to be a

complete resolution of cognitive impairment,

although social functioning can take longer to

improve 
Loneliness is the most significant aetiological It's under-recognised and difficult to measure factor for both cognitive dysfunction and depression

Most antidepressants don't directly improve cognition. I think it would be useful for trials to look at the effect of antidepressants on cognitive function specifically, as well as changes in mood. I am not aware of any such data. I think this would be key in deciding what agent to use in certain cases

It's the Cinderella of medicine mental health
It's difficult to say that there are persistent cognitive symptoms after remission of depression. There is gradual recovery even in case of severe depression, though some patients have memory lapse of the acute episode

\section{Detection}

Most patients over the age of 55 years have multiple co-morbidities, which often cause depression and reduced cognitive function It's a sapping process dealing with mental health and we haven't the time as primary care physicians

No. I'm aware of cognitive dysfunction in depression as part of symptoms depression may present with; the PHQ9 score doesn't really assess this
It is worth investigating for dementia in older patients presenting with cognitive impairment and depression for the first time There are no systematic ways of assessing cognitive function other than referral for neuropsychology testing, which is not feasible given current resources More questionnaires won't help patients especially when there is cognitive impairment. I am sceptical of forcing cognitive dysfunction into the limelight to create a marketing niche 
Although it is subjective and not really

formally assessed, it is common sense and the symptoms tie in with depression although

other causes need to be excluded also

In early dementia, MDD has high prevalence

Do have screening tools available but don't concentrate just on cognitive function. Also am limited with what I can do in a 10-minute consultation

I often diagnose depression in patients with memory impairment or other cognitive difficulties. This is largely based on history rather than a formal objective assessment
There is good understanding of cognitive dysfunction by secondary care professionals (some health-care support staff do not understand and need some education about it). Patients and relatives express concerns about cognitive dysfunction

Cognitive symptoms often go unrecognised We need access to good-quality screening and in-depth assessment tools

I am not convinced that routine cognitive assessment is appropriate for all patients with depression: existing tools may be too complex/strenuous. Sometimes, it is also more beneficial to await the resolution of depressive symptoms to avoid denting a patient's confidence when it is already fragile

Is clearly linked in older people with depression - but are we forgetting it in the younger severely depressed population? Depression can have subtle or no psychological symptoms 


\section{Management}

I've found some patients with "chronic depression" often have associated impaired or reduced cognitive function. I don't know which causes which

Validated tools for use in primary care would be useful in assessing/detecting and then monitoring cognitive function. The only tools we have are designed primary for dementia-related illness

The combination of CBT with antidepressants tend to work for most patients; however, there is a need to reassess cognitive dysfunction at least twice a year for patients with major depressive disorder

These patients will usually be under the care of the community psychiatry team. In my area there does not seem to be much communication regarding cognitive dysfunction in these patients, certainly not longer-term monitoring

\author{
Although poor concentration is a core \\ symptom of depression which is often \\ enquired about, other cognitive domains are \\ not commonly tested for/addressed
} I would like to learn more about the evidence about exercise, lifestyle changes, etc. This is an area that needs to be focused on more in clinical practice to improve the quality of life of the patients

Cognitive function should be reassessed following improvement of depressive symptoms. If impairment persists should investigate further for dementia

The effects of lifestyle, behavioural activation, exercise, etc are mediated by improvement in depression and therefore concentration. Formal testing is either blunt (bedside - MoCA/MMSE, etc) or extremely expensive, with a waiting list longer than the average episode of depression. The important thing is finding out whether the concentration and memory problems are affecting ability to drive, work, care or parent 
In general practice, we do not have the luxury In the absence of evidenced treatments, of following up a patient 4-6 months after assessments (even if they were available) are they have ceased taking antidepressants difficult to prioritise. Which is a shame Although exercise or cognitive training and There is role for functional neuroimaging too CBT may help with depression, I am in establishing the link between depression uncertain of the evidence in relation to these and cognitive deficits

Abbreviations: GP, general practitioner; PHQ9, Patient Health Questionnaire;

MDD, major depressive disorder; CBT, cognitive behavioural therapy;

MoCA, Montreal Cognitive Assessment; MMSE, Mini Mental State Examination. 


\section{Figure legends}

Fig. 1. Statements on burden of cognitive dysfunction in depression (A) and responses (B).

Abbreviations: GP, general practitioner; Psych, psychiatrist.

Fig. 2. Statements on detection of cognitive dysfunction in depression (A) and responses (B).

Abbreviations: GP, general practitioner; Psych, psychiatrist.

Fig. 3. Statements on management of cognitive dysfunction in depression (A) and responses (B).

Abbreviations: GP, general practitioner; Psych, psychiatrist. 